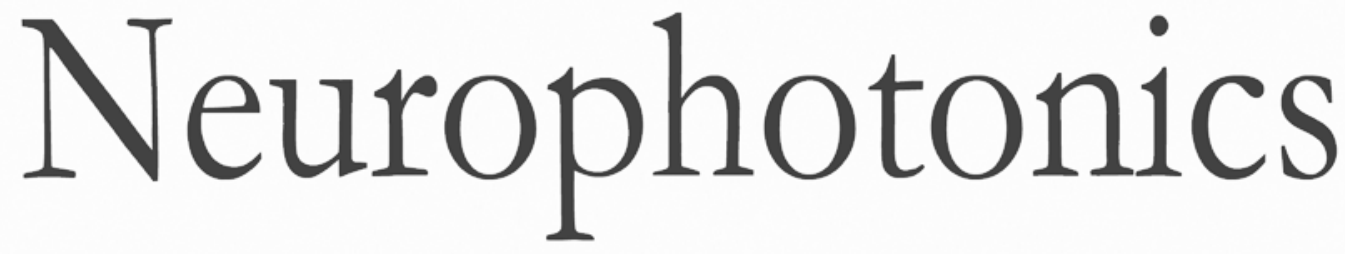

\title{
Pioneers in Neurophotonics: Special Section Honoring Professor Amiram Grinvald
}

Ron D. Frostig

Carl C.H. Petersen 


\title{
Pioneers in Neurophotonics: Special Section Honoring Professor Amiram Grinvald
}

\author{
Ron D. Frostig \\ University of California Irvine \\ Departments of Neurobiology and Behavior and Biomedical Engineering \\ Irvine, California, United States
}

Carl C.H. Petersen

Laboratory of Sensory Processing

Brain Mind Institute, Faculty of Life Sciences

Ecole Polytechnique Federale de Lausanne (EPFL)

$\mathrm{CH}-1015$ Lausanne, Switzerland

Amiram Grinvald is a leading pioneer of functional brain imaging. In a career that has spanned over 40 years, he has contributed importantly to neuroscience by developing new optical methods that have revealed novel insights into brain function.

Amiram was first introduced to recording neuronal changes in membrane potential based on voltage sensitive dyes (VSD) during his postdoctoral years (1975-1978) in the laboratory of Larry Cohen at Yale University. At that time, the technique was mainly applied to single neurons and their processes. Together with colleagues in Cohen's laboratory, Amiram helped expand the application of VSD techniques to simultaneously probe the electrical activity of many neurons at a period when neurons were typically probed oneat-a-time with a single microelectrode. Following his postdoctoral training, he established his own laboratory at the Weizmann Institute of Science in Israel to start an outstanding research career as a pioneer of in vivo optical imaging (a term he coined in a 1984 paper).

Amiram's career involved a continuous drive for pushing the envelope on technical developments that, in turn, led to unprecedented findings. Together with his colleagues, he made many important advances, including the continuous development of new, better voltage-sensitive dyes based on his long collaboration with Rina Hildesheim; the construction of new camera systems with increasing spatiotemporal capabilities; the development of cranial window systems that allowed for chronic imaging and electrode recording in anesthetized cats, anesthetized monkeys and, importantly, in behaving primates; the combination of microstimulation and VSD imaging (VSDI) in awake monkeys; the development of imaging spectroscopy and oxygen tension measurements in cortex; and the development of blood flow velocity measurements in small blood vessels. Along with his research team, he achieved another major technological development by discovering intrinsic signal optical imaging (ISOI), a high spatial resolution technique that enabled functional imaging of cortical activity patterns through a thinned skull. Using only light without applying any exogenous substances, this technique enabled for the first time mapping of several types of columnar systems underlying the same

C 2017 Society of Photo-Optical Instrumentation Engineers (SPIE) large cortical area and study of their relationships first in anesthetized and later in awake behaving animals.

These technical developments led to important findings that repeatedly appeared in major scientific journals and are still highly cited. Among them are the discovery of the functional architecture of several cortical areas at the cortical column level; the discovery that evoked subthreshold activity spreads far beyond retinotopic borders; the discovery of the large effects of internal ongoing (spontaneous) cortical activity on sensory processing and conversely of sensory processing on ongoing activity; revealing the neurovascular interactions between evoked activity and cortical microcirculation; and the application of ISOI as an ophthalmic diagnostic medical tool. Indeed, Amiram was a major figure in unraveling cortical functional architecture at the mesoscopic level - the level of large ensembles of activated, interacting neurons. Together, his findings helped to launch the careers of many of his students and collaborators in universities and research institutes around the world.

Amiram spent several years (1985-1991) as a visiting professor in Nobel laureate Torsten Wiesel's laboratory at Rockefeller University in New York. Here is a tribute to Amiram's accomplishments contributed by Wiesel for the current Neurophotonics special section:

\footnotetext{
"Amiram Grinvald spent close to five years in my laboratory working on the optical imaging of the columnar organization of the cat and monkey visual cortex. With help of his intrinsic signals and voltage sensitive dye imaging techniques, we suddenly could see in vivo the columns of cells with the same preference of orientation and of ocular dominance. In the field of vision his method was a breakthrough soon used in labs all over the world. New methods open doors, and Amiram has contributed to many new discoveries and insights about the neural circuits and interactions in the mammalian visual cortex.
}

Amiram has a well-defined profile of stubborn determination bringing his often-brilliant ideas against any theoretical or practical hindrances to successful conclusions. The world of research can be a lonely place, but his success is no doubt due to his amazing energy, imagination and reach for the apparently impossible. During my long life as a neuroscientist I have had the privilege and pleasure to meet many outstanding colleagues and Amiram Grinvald is one of these individuals." 
On a personal level, Amiram has always been highly energetic and focused on research, constantly wishing to run the next experiment. He has always been "hands-on," highly involved in every experiment. Characteristically, the potential difficulty of the next experiment was never a consideration but a challenge to overcome, and sleep and food were always optional, especially when cigarettes were available ...

This special section of Neurophotonics includes research papers that serve as a reminder for the continuing impact of Amiram's pioneering research in several of the research topics that he helped launch and establish.

Ron Frostig, PhD, is a professor of neurobiology and behavior at University of California, Irvine. His major research interests include the structure, function, and plasticity of sensory neocortex, which he studies by employing functional imaging, electrophysiological, and anatomical techniques in rodents. He was trained in electrophysiology by Moshe Abeles (Hebrew University, Jerusalem) and Ron Harper (UCLA, Los-Angeles), and in developing and applying functional imaging methods by Amiram Grinvald and Torsten Wiesel (Rockefeller University, New York).

Carl Petersen is professor of neuroscience at the Ecole Polytechnique Federale de Lausanne (Switzerland), where he investigates sensory perception in mice using electrophysiological and optical techniques. After investigating calcium signaling during his $\mathrm{PhD}$ in Cambridge (UK) supervised by Prof. Sir Michael Berridge, he trained in neurophysiology with Prof. Roger Nicoll at the University of California San Francisco (USA) and Prof. Bert Sakmann at the Max Planck Institute for Medical Research in Heidelberg (Germany). 